\title{
ORIGINAL ARTICLE \\ Isolation, identification, and selection of strains as candidate probiotics and starters for fermentation of Swedish legumes
}

\author{
Inger-Cecilia Mayer Labba'*, Thomas Andlid', Åsa Lindgren², Ann-Sofie Sandberg' \\ and Fei Sjöberg ${ }^{3}$ \\ 'Division of Food and Nutrition Science, Department of Biology and Biological Engineering, Chalmers University \\ of Technology, Gothenburg, Sweden; 2Department of Clinical Bacteriology, Institute of Biomedicine, Sahlgrenska \\ Academy, University of Gothenburg, Gothenburg, Sweden; ${ }^{3}$ Department of Infectious Diseases, Institute of \\ Biomedicine, Sahlgrenska Academy, University of Gothenburg, Gothenburg, Sweden
}

\section{Popular scientific summary}

- Demand for fermented non-dairy products is increasing, there is a need to develop adapted starter cultures.

- We isolated 25 lactic acid bacteria strains from legumes. The strains were characterized for potential probiotic properties and tested for their ability to ferment a bean beverage.

- Pediococcus strains could grow in bean beverage and improve bioavailability of minerals in co-fermentation with yeast.

- Results indicate that new and improved fermented non-dairy products could be developed.

\section{Abstract}

Background: The non-dairy sector is growing, fermented alternatives to dairy are sparse. Adapted starter cultures to substituting raw materials needs to be developed.

Objective: Aims of this study were to isolate, identify, and phenotypically characterize lactic acid bacteria (LAB) that inhabit Swedish legumes, and assess properties necessary for selecting strains with the ability to ferment a bean beverage and with potential health beneficial properties.

Design: Isolates of presumed LAB were obtained from legumes collected at Öland, Sweden. Strain diversity was assessed by repetitive polymerase chain reaction (rep-PCR). The strains were identified using matrix-assisted laser desorption/ionization-time-of-flight mass spectrometry (MALDI-TOF MS). Species belonging to Enterococcus were predominant along with Pediococcus and closely related Bacillus. Strains were tested for tolerance to low pH, phenol, and bile as well as their bile salt hydrolase (BSH) activity. In addition, Enterococcus strains were tested for antibiotic resistance, and Pediococcus strains for their ability to ferment a bean beverage.

Results: From the 25 strains characterized, five were found resistant to low $\mathrm{pH}$, bile, and phenol, suggesting that they can survive a passage through the gastrointestinal tract (GIT) and hence potentially exert beneficial effects in the host. These are suggested for further investigation on specific host-beneficial properties. Two of these, belonging to Pediococcus pentosaceus, were able to ferment a bean beverage without any added nutrients, indicating that the Pediococcus strains are well adapted to the bean substrate. One of the P. pentosaceus strains were also able to markedly improve the reduction of phytate by the phytase-producing yeast strain Pichia kudriavzevii TY1322 during co-fermentation as well as increase the final cell count of the yeast strain. Conclusion: Strain isolation and characterization performed in this study aids in selecting starter cultures for legume fermentation. Nutritional properties can be improved by co-fermentation with yeast indicating that novel nutritious fermented non-dairy products could be developed.

Keywords: isolation; fermentation; fava bean; pediococcus pentosaceus; phytate 
$\mathrm{P}$ ulses, such as beans and lentils, have a favorable nutritional composition with high contents of protein, dietary fibers, minerals, vitamins, and phytochemicals, and positive health effects have been demonstrated (1). Furthermore, pulses have been suggested as alternatives to resource-demanding animal products, and could contribute to climate change mitigation by lowering greenhouse gas emissions in arable systems (2).

Foods with a low environmental impact need to become more accessible, economically sustainable, and attractive for both farmer and consumer. In the Scandinavian countries, where cultivation of soy is not suited to the climate, other domestic protein crops, such as fava beans, have a large potential in partly replacing animal products. This raises the need to develop new palatable food products with low environmental impact. Fermentation is a well-known process with low energy use that can provide several positive nutritional and sensory properties to raw materials such as grain legumes $(3,4)$. Depending on the organisms used, the end product will have different properties and nutritional values (4). Previous studies have shown that certain microorganisms can synthesize vitamins such as folic acid, riboflavin, niacin, thiamin $(5,6)$, and B12 (7) using grain legumes as a substrate. Some bacteria are able to metabolize at least two of the main components (n-hexanal and pentanal) that give a bean-like flavor to soy milk (8). Furthermore, fermentation lowers the level of oligosaccharides, which can cause postprandial flatulence from legumes $(9,10)$, and other anti-nutritional components such as tannins, phytate (11) , and trypsin inhibitors (12). Different strains within the same species can have completely distinct metabolic patterns, which, in turn, affect not only synthesis of vitamin and breakdown of undesirable components but also production of substances that affect taste and texture of the product, such as exopolysaccharides (13).

Lactic acid bacteria, LAB, are a vast group of bacteria within the phylum Firmicutes and the class Bacilli, showing considerable genotypic and phenotypic diversity. The closely related genus Bacillus belongs to the same class (Bacilli) and hence related to LAB. Both LAB and Bacillus include probiotic as well as pathogenic organisms. LAB are the most used bacteria within the food sector and many strains have been graded as Generally Recognized as Safe (GRAS) strains. Despite generally not being able to reduce the levels of phytate, LAB can provide favorable conditions for phytase activity and contribute indirectly to an improved phytate reduction important to improve bioavailability of essential dietary minerals, such as iron and zinc, in cereals and legumes (14). Co-fermentation between LAB and phytase-producing yeast strains takes place in many natural food fermentations $(15,16)$. Cofermentation between LAB and yeast has been suggested to improve sensory properties and prolong shelf life as well as improve resistance to spoilage molds (17). This indicates interesting applications within the food industry for new strains of LAB.

Despite already extensively used in the food industry, there is still a need to find and develop starter cultures functionally adapted to different types of plant-based raw material as alternative to dairy products. Apart from the functional and nutritional improvements during fermentation, LAB can also have probiotic properties. According to the World Health Organization (WHO), probiotics are 'live microorganisms which, when administrated in adequate amounts, confer a health benefit to the host' (18). Health benefits include reduction in hypercholesterolemia (19), reduction in symptoms of irritable bowel syndrome (20, 21), normalization of disturbed gut microbiota, regulation of intestinal transit, competitive exclusion of pathogens, and production of short chain fatty acids (22). The importance of gut microbiota in health and disease has become increasingly evident, and is reflected in the popularity of fermented and probiotic foods among consumers and their rapid market growth (23). The gut microbiota can be modified by the food consumed, for instance by dietary fiber present in legumes, as well as live microbes present in fermented food (24). New probiotics should be selected based on their strain characteristics. The main selection criteria for probiotics include many functional properties, such as tolerance to gastric acidity, bile, and phenol toxicity and ability to de-conjugate bile salts (25). In the present study, we searched for, and initiated studies of, strains that efficiently metabolize complex carbohydrates in pulses, with the long-term goal to develop new types of digestible, palatable, and nutritious fermented legume-based foods.

The aim was to identify and isolate strains with the ability to ferment a fava bean beverage and to characterize LAB isolated from Swedish grain legumes, perform co-fermentation to reduce phytate, a major inhibitor of iron and zinc absorption, and initial test of probiotic potential of the strains.

\section{Materials and methods}

\section{Plant samples}

A total of 23 samples were collected from fields $(n=12)$ and from stored seeds $(n=11)$ derived from plants cultivated during 2015. The 12 field samples came from five different locations of Öland, Sweden. On each site, approximately $50 \mathrm{~g}$ were collected by picking whole pods directly from the plant with sterile gloves. Samples were placed in clean bags and stored at $4^{\circ} \mathrm{C}$ until they were used for analysis. The remaining 11 samples were seeds that were harvested at Öland, dried and stored at $4^{\circ} \mathrm{C}$ for 8 months by the producer. The samples were different varieties of Phaseolus vulgaris (black beans, brown beans, kidney beans, navy beans, borlotti beans, and yin yang), and two varieties of Pisum sativum (grey and yellow peas). 


\section{Isolation of $L A B$}

For the selection of LAB from the bean samples, about $1 \mathrm{~g}$ of bean pods were aseptically transferred to de Man, Rogosa and Sharpe (MRS) broth (Sigma-Aldrich, Missouri, USA) with $\mathrm{pH}$ adjusted to 5.7 using $\mathrm{HCl}$ (SigmaAldrich). The flasks were incubated at $37^{\circ} \mathrm{C}$ for 4 days in a shaking incubator (Barnstead Lab-Line, ON, Canada) at $190 \mathrm{rpm}$. Dilutions of the liquid were spread in parallels on MRS agar plates (Scharlab, Spain); these were then incubated at anaerobic conditions using Anaerocult ${ }^{\circledR}$ A (Merck Millipore, Germany) at $37^{\circ} \mathrm{C}$ for $72 \mathrm{~h}$. Colonies were picked out and re-streaked on MRS agar plates until isolated bacterial strains were obtained. Strain isolation was made for two subsequent times for each sample.

\section{Identification of isolates}

Colonies that were Gram-positive, oxidase-negative, and catalase-negative were selected for further analyses. These isolates were harvested for DNA extraction and stored at $-80^{\circ} \mathrm{C}$.

\section{Repetitive polymerase chain reaction (rep-PCR) genomic} fingerprinting

To minimize the risk of duplicate strains, we performed rep-PCR. Genomic DNA was extracted from 116 isolates according to a previously described protocol (26). One loop of bacteria incubated at $39^{\circ} \mathrm{C}$ for $74 \mathrm{~h}$ on horse blood agar plates (Scharlab) were inoculated in $50-\mu \mathrm{L}$ lysis buffer (10 mmol/L Tris $\mathrm{HCl}, 1 \mathrm{mmol} / \mathrm{L}$ EDTA, and $10 \mathrm{mmol} / \mathrm{L}$ saline; Sigma-Aldrich) and incubated for $10 \mathrm{~min}$ at $95^{\circ} \mathrm{C}$. Samples were then centrifuged for $5 \mathrm{~min}$ at $12,000 \times \mathrm{g}$. The supernatant was collected, measured for DNA concentration using a NanoDrop 2000c spectrophotometer (Thermo Fisher Scientific, Massachusetts, USA), and stored at $-20^{\circ} \mathrm{C}$ until used.

Strain diversity was assessed by rep-PCR using the single nucleotide (GTG) 5 primer (5'-GTGGTGGTGGTGGTG-3') (Invitrogen, California, USA) (27). Reactions were performed using 2,720 thermal cycler (Applied Biosystems, California, USA). The amplification program had an initial denaturation of $98^{\circ} \mathrm{C}$ for $3 \mathrm{~min}$, followed by 30 cycles of denaturation $\left(98^{\circ} \mathrm{C}\right.$, $30 \mathrm{~s})$, annealing $\left(40^{\circ} \mathrm{C}, 1 \mathrm{~min}\right)$, extension $\left(65^{\circ} \mathrm{C}, 8 \mathrm{~min}\right)$, and a final single extension $\left(65^{\circ} \mathrm{C}, 16 \mathrm{~min}\right)$. Each $25 \mu \mathrm{L}$ of PCR reaction contained $1 \mathrm{x}$ HF Phusion PCR buffer (Thermo Fisher), $2.5 \mathrm{mmol} \mathrm{MgCl}_{2}$ (Thermo Fisher), $170 \mu \mathrm{g} / \mathrm{mL}$ BSA (Thermo Fisher), $200 \mu \mathrm{mol} / \mathrm{L}$ dNTPs (Thermo Fisher), $2 \mu \mathrm{mol} / \mathrm{L}$ (GTG) ${ }_{5}$ primer (Invitrogen), $0.05 \mathrm{U}$ Phusion polymerase (Thermo Fisher), 5\% Dimethyl sulfoxide (DMSO; Thermo Fisher), and $60 \mathrm{ng}$ of template DNA.

Amplification products were resolved by electrophoresis on a $25-\mathrm{cm}$ gel in a $40-\mathrm{cm}$ cell (Bio-Rad, Sub Cell GT, California, USA) at a constant voltage of $1.5 \mathrm{~V} / \mathrm{cm}$ for $16 \mathrm{~h}$ in $4-\mathrm{mm} 1.5 \%(\mathrm{w} / \mathrm{v})$ agarose (Thermo Fisher) gel in 1x TBE buffer (Sigma-Aldrich). A DNA molecular weight ladder (Thermo Fisher) was used as a reference. Gels were stained post-run with $3 x$ Gelred in $0.1 \mathrm{M} \mathrm{NaCl}$ (Biotium, California, USA) and visualized by UV light using a Gel Doc (BioRad).

The PCR fingerprints were analyzed using BioNumerics (Applied Maths NV, Sint-Martens-Latem, Belgium). Similarity of digitalized band patterns was calculated using the Sørensen-Dice coefficient. Complete linkage algorithms were used to construct an average linkage dendrogram to show relationship of isolates.

\section{MALDI-TOF MS identification of isolates}

The 116 bacterial isolates were analyzed twice with matrix-assisted laser desorption ionization-time-of-flight mass spectrometry (MALDI-TOF MS) analysis using intact cell biomass obtained from overnight cultures of $\mathrm{LAB}$ at $37^{\circ} \mathrm{C}$ on horse blood agar plates. Samples were spotted in duplicates on disposable target plates (bioMeriéux, Marcy l'Etoile, France) and overlaid with $1 \mu \mathrm{L}$ VITEK $^{\circledR}$ MS-FA (Formic acid; bioMeriéux), then air-dried and again overlaid with $1 \mu \mathrm{L}$ VITEK $^{\circledR}$ MSCHCA matrix solution ( $\alpha$-cyano-4-hydroxycinnamic acid; bioMeriéux). MALDI-TOF MS analysis was performed using a VITEK ${ }^{\circledR}$ MS instrument (bioMeriéux) in the mass range of $2,000-20,000 \mathrm{~m} / \mathrm{z}$, and the spectral data were analyzed using the VITEK ${ }^{\circledR}$ MS IVD and VITEK ${ }^{\circledR}$ MS SARAMIS/RUO databases (bioMeriéux).

\section{Phenotypic tests related to probiotic potential}

A number of prerequisites need to be fulfilled for a strain to be considered a candidate for further investigation of its probiotic properties. We studied the ability of strains to survive the harsh conditions such as in the gastrointestinal tract (GIT), including tolerance to bile, low $\mathrm{pH}$, and phenol.

\section{Tolerance to low $\mathrm{pH}$}

Survival of strains in acidic conditions was asessed by incubation in low $\mathrm{pH}$ growth media according to a previously described method (28) using $\mathrm{pH}$ 2.5. Unique strains, which we identified by MALDI-TOF, were propagated twice in MRS broth for $24 \mathrm{~h}$ at $37^{\circ} \mathrm{C}$, collected by centrifugation (at 5,000 $\times \mathrm{g}$, for $5 \mathrm{~min}$ ), and washed twice in phosphate buffer solution (PBS; Thermo Fisher). After propagation and dilution, cells were inoculated in triplicates into $8 \mathrm{~mL}$ of MRS broth adjusted to pH 2.5 to simulate gastric conditions. Cells were then incubated at $37^{\circ} \mathrm{C}$ for $2.5 \mathrm{~h}$ with continuous stirring using a tube rotator (New Brunswick Scientific, New Jersey, USA). Bacterial enumeration was performed using five replicates per dilution on MRS agar plates at $0,0.5,1$, $1.5,2$, and $2.5 \mathrm{~h}$. 


\section{Phenol tolerance}

The isolates' ability to survive in the presence of phenol, which can be present in the gut, was examined according to a previously described method (29), with minor modifications. Unique strains were prepared in the same way as described for the low $\mathrm{pH}$ test. The $\mathrm{OD}_{600}$ was adjusted to $0.4-0.6$, then $200 \mu \mathrm{L}$ of the diluted cells were inoculated in duplicates into $8 \mathrm{~mL}$ of MRS broth, $\mathrm{pH} 5.7$, with $0.4 \%$ phenol (Sigma Aldrich). The cells were then incubated in a shaking incubator at $37^{\circ} \mathrm{C}$ for $24 \mathrm{~h}$. Bacterial enumeration was performed using five replicates per dilution on MRS agar plates at 0 and $24 \mathrm{~h}$.

\section{Bile tolerance}

Tolerance of the isolates to bile salts was assayed as described by Gilliland et al. with slight modifications (30). Prior to the bile tolerance test, strains were prepared in the same way as described for the low $\mathrm{pH}$ test. The $\mathrm{OD}_{600}$ was then adjusted to $0.4-0.6$ with PBS, and $200 \mu \mathrm{L}$ of the diluted cells were inoculated in duplicates into $8 \mathrm{~mL}$ of MRS broth with $0.3 \%$ (w/v) bile salts (Sigma Aldrich). Cells were then incubated in a shaking incubator at $37^{\circ} \mathrm{C}$ for $48 \mathrm{~h}$. The bacterial suspension was assessed as colony forming units/milliliter $(\mathrm{CFU} / \mathrm{mL})$ using five replicates on MRS agar plates at 0, 24, and $48 \mathrm{~h}$.

Qualitative determination of bile salt hydrolase (BSH) activity The taurodeoxycholic acid (TDCA) hydrolase activity was determined for unique strains using $0.5 \%(\mathrm{w} / \mathrm{v})$ bile salt-MRS agar plate assay (31). MRS agar plates were supplemented with 0.5\% TDCA (Sigma Aldrich).

Bile salt-MRS agar plates were plated in duplicates with each representative strain and incubated anaerobically using an Anaerocult ${ }^{\circledR} \mathrm{A}$ at $37^{\circ} \mathrm{C}$ for $72 \mathrm{~h}$ before observation. A precipitation zone surrounding colonies indicated BSH activity.

\section{Screening of isolated Enterococcus strains for antibiotic resistance}

Isolates identified as Enterococcus species were tested for antibiotic resistance using the disk diffusion test according to the Clinical and Laboratory Standards Institute (CLSI) (32). The isolated Enterococcus was tested for resistance to ampicillin, trimethoprim, vancomycin, and nitrofurantoin.

\section{Production of bean flour}

Dried fava beans were soaked overnight in deionized water with $2 \%(\mathrm{w} / \mathrm{v}) \mathrm{NaHCO}_{3}$ (Sigma Aldrich), then peeled by hand and incubated at $95^{\circ} \mathrm{C}$ in deionized water with $0.5 \%$ (w/v) $\mathrm{NaHCO}_{3}$ for $4 \mathrm{~min}$ in a water bath. The boiled beans were then blended in $90^{\circ} \mathrm{C}$ deionized water for $60 \mathrm{~s}$ and put on an ice bath to cool before freeze-drying. The freeze-dried flour was then stored at $-18^{\circ} \mathrm{C}$ until use.

\section{Fermentation of fava bean flour medium by} Pediococcus pentosaceus

A bean flour medium was prepared using deionized water with $6 \%(\mathrm{w} / \mathrm{v})$ fava bean flour brought to a boil, with $\mathrm{pH}$ adjusted to 6.2 , filtered through a cheese cloth and autoclaved. Selected strains belonging to Pediococcus pentosaceus were pre-cultured overnight in liquid MRS at $37^{\circ} \mathrm{C}$ in a shaking incubator. The cells were washed twice in PBS and diluted in PBS to an $\mathrm{OD}_{600}$ of $0.5-0.6$. In triplicates, $8 \mathrm{~mL}$ of the fava bean medium were inoculated with $200 \mu \mathrm{L}$ of diluted cells from five selected strains. Bacterial enumeration was performed using five replicates per dilution on MRS agar plates at $0,4,6,8,12,24$, and $48 \mathrm{~h}$.

\section{Co-fermentation of fava bean flour medium}

Bean flour medium was prepared as described for fermentation by $P$. pentosaceus. Pichia kudriavzevii TY1322 (33) and P. pentosaceus strain 77 were pre-cultured overnight in liquid MRS and yeast extract peptone dextrose (YPD) medium respectively at $37^{\circ} \mathrm{C}$ and $220 \mathrm{rev} / \mathrm{min}$ in a rotary shaker. Cells were washed twice in PBS and diluted to an $\mathrm{OD}_{600}$ of $0.55-0.60$. In triplicates, $80 \mathrm{~mL}$ of the fava bean medium were inoculated in three combinations: (1) $600 \mu \mathrm{L}$ of diluted yeast cells $+600 \mu \mathrm{L}$ of diluted bacterial cells, (2) $600 \mu \mathrm{L}$ of diluted bacterial cells, and (3) $600 \mu \mathrm{L}$ of diluted yeast cells. Microbial enumeration was performed using five replicates per dilution on agar plates at $0,4,6,8,12,24,36,48 \mathrm{~h}$. YPD agar plates with $100-\mathrm{mg} / \mathrm{L}$ chloramphenicol (Sigma-Aldrich) were used for combinations 1 and 3. MRS agar plates with $0.5-\mathrm{mg} / \mathrm{mL}$ cycloheximide (Sigma-Aldrich) were used for combination 1 and 2. Each time, set microbial enumeration was performed and samples for phytic acid analysis were withdrawn from the fermentation media.

\section{Phytate analysis}

Fava bean flour medium $(500 \mu \mathrm{L})$ was collected during fermentation and immediately mixed with $500 \mu \mathrm{L}$ of 1 $\mathrm{M} \mathrm{HCl}$ to stop enzymatic and microbial activity. Samples were stored at $-20^{\circ} \mathrm{C}$ until analysis. Samples were thawed and mixed using a laboratory shaker (Heidolph Reax 2; Heidolph Instruments $\mathrm{GmbH}$, Schwabach, Germany) for $3 \mathrm{~h}$, centrifuged, and the supernatant was transferred to an HPLC vial. Phytate was analyzed as inositol hexaphosphate ( $\operatorname{InsP}_{6}$ ) by high-performance ion chromatography (HPIC) according to Carlsson et al. (34). The chromatography setup consisted of an HPLC pump (model PU-4080i; Jasco Inc., Easton, MD, USA) for the eluent and an RHPLC pump (model PU-4180; Jasco) equipped with a PA-100 guard column and a CarboPac PA-100 column.

InsP $_{6}$ was eluted with an isocratic eluent of $80 \% \mathrm{HCl}$ (1 M) and $20 \% \mathrm{H}_{2} \mathrm{O}$ at $0.8 \mathrm{~mL} / \mathrm{min}$, subjected to a post- 
column reaction with ferrous nitrate, and detected with at $290 \mathrm{~nm}$ in a UV-visible HPLC detector (UV-4075; Jasco). Each sample had a run time of $7 \mathrm{~min}$, and the $\mathrm{InsP}_{6}$ concentration was calculated using external standards covering the concentration range of $0.1-0.6 \mu \mathrm{M} / \mathrm{mL}$.

\section{Results}

\section{Isolation and identification by Rep-PCR and MALDI-TOF MS}

A total of 116 isolates of putative LAB were originally obtained from 23 legume samples. Strains fulfilling all criteria used to select LAB and closely related lactateproducing bacteria were isolated from each legume, except from the samples of black beans, from which we could not isolate any LAB. Using rep-PCR fingerprints, the 116 isolates were clustered into five main groups. Of these, fingerprints showing $>90 \%$ similarity were assigned to the same genotype and were considered a cluster, as suggested by Maluping et al. (35).

From each cluster, one strain was randomly picked as a representative of the group for further analysis, resulting in a total of 25 unique strains (Fig. 1). MALDI-TOF MS identified the strains as Enterococcus hirae, Enterococcus faecium, Enterococcus mundtii, Bacillus coagulans, and P. pentosaceus.

The collected borlotti beans were found to have the highest LAB diversity, with strains from almost all identified genera present. Conversely, no LAB was isolated from black beans despite repeated enrichments. In total, eight unique strains of $P$. pentosaceus and six unique strains of $B$. coagulans were found from the plant samples (Table 1).

\section{Phenotypic tests related to probiotic potential}

\section{Tolerance to low $\mathrm{pH}$}

Each of the unique B. coagulans strains showed a high degree of tolerance to $\mathrm{pH} 2.5$ and had an exponential growth during the entire incubation (Fig. 2A). Strains belonging to E. faecium and E. mundtii showed a generally low tolerance to $\mathrm{pH} 2.5$ and were completely depleted after incubation (Figs 2B and 2C). P. pentosaceusstrains (Fig. 2D) showed two distinctive growth patterns; strain number 77, 100, 101, and 176 showed tolerance to low $\mathrm{pH}$ with small variations of CFU/mL. On the contrary, P. pentosaceus strains 96, 97, and 177 did not survive incubation (Fig. 2D).

\section{Phenol tolerance}

Overall, strains from all genera showed tolerance to $0.4 \%$ phenol with a few exceptions. These were $B$. coagulans strains 222 and 224 (Fig. 3A) and E. mundtii strain 94

Table 1. Isolated strains of LAB and Bacillus and their origin

\begin{tabular}{llc}
\hline Species & \multicolumn{1}{c}{ Isolated from } & $\begin{array}{c}\text { Number of } \\
\text { unique strains }\end{array}$ \\
\hline Bacillus coagulans & $\mathrm{KB}^{* *}, \mathrm{YY}^{*}$ & 6 \\
Enterococcus hirae & $\mathrm{BoB} \ddagger$, GP§ & 2 \\
Enterococcus faecium & $\mathrm{BoB}, \mathrm{YPT}, \mathrm{KB}, \mathrm{WB} \dagger$ & 3 \\
Enterococcus mundtii & $\mathrm{BoB}, \mathrm{BB} \dagger$ & 6 \\
Pediococcus pentosaceus & $\mathrm{YY}, \mathrm{BB}, \mathrm{BoB}$ & 8 \\
Total number of strains & 25 & - \\
\hline
\end{tabular}

*YY = yin yang beans; $\nmid \mathrm{BB}=$ brown beans; $\ddagger \mathrm{BoB}=$ borlotti beans; $\S G P=$ grey peas; $\mathbb{T Y P}=$ yellow peas, ${ }^{* *} \mathrm{~KB}=$ kidney beans; $\dagger+\mathrm{W} B=$ white beans.

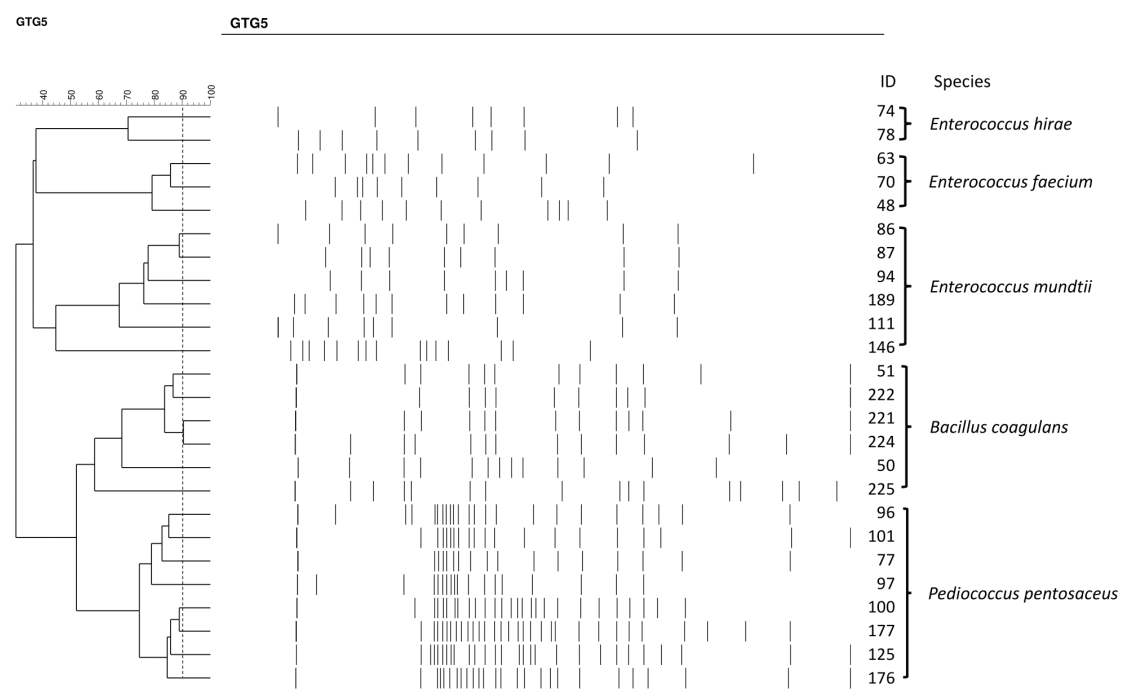

Fig. 1. Dendrogram generated after cluster analysis of the digitalized (GTG) $)_{5}$ PCR fingerprints of the isolated strains. Cluster analysis was made using the Sørensen-Dice coefficient and a complete linkage algorithm. 

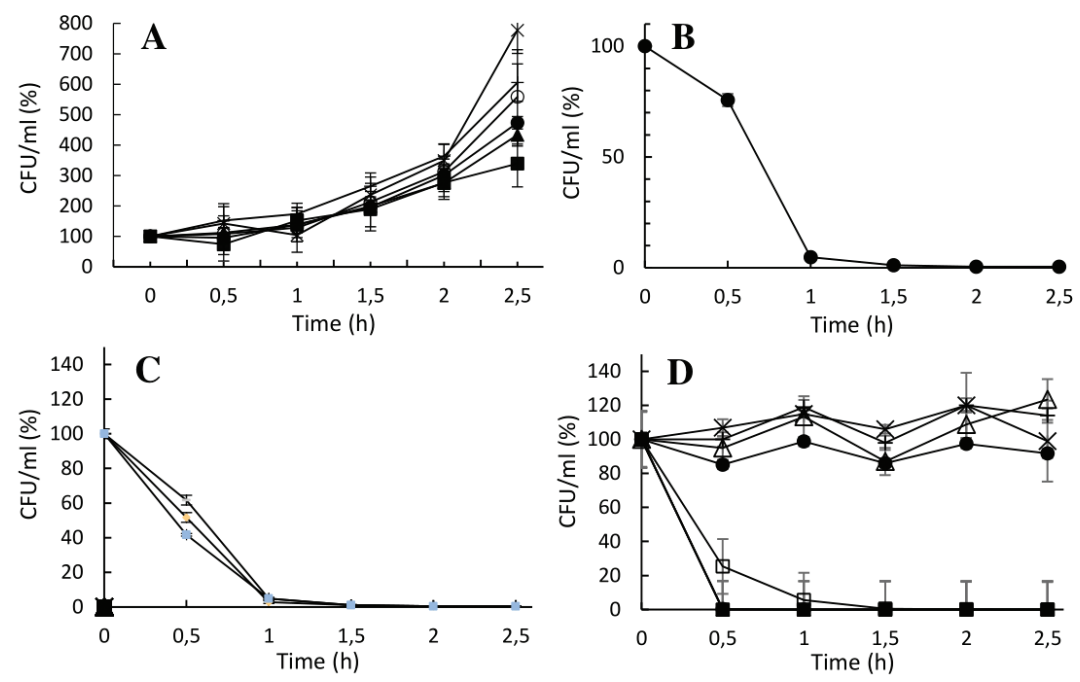

Fig. 2. Cell concentration as a function of incubation time during low pH stress ( $\mathrm{pH} 2.5)$ in MRS. Data presented as percentage of the initial CFU/mL (set as 100\%). Graph A: B. coagulans; graph B: E. faecium; graph C: E. mundtii; and graph D: P. pentosaceus. Symbols correspond to different strain numbers. Graph A, B. coagulans: $50(\bullet), 51(\mathbf{\Delta}), 221(\boldsymbol{\bullet}), 222(\times), 224(+)$, 225 (०); graph B, E. faecium: $63(\bullet)$; graph C, E. mundtii: $94(\bullet), 146(\boldsymbol{\Delta}), 189(\bullet)$; and graph D, P. pentosaceus: $77(\bullet), 96(\mathbf{\Delta})$, $97($ (), $100(\times), 101(+), 125(\circ), 176(\Delta), 177(\square)$.
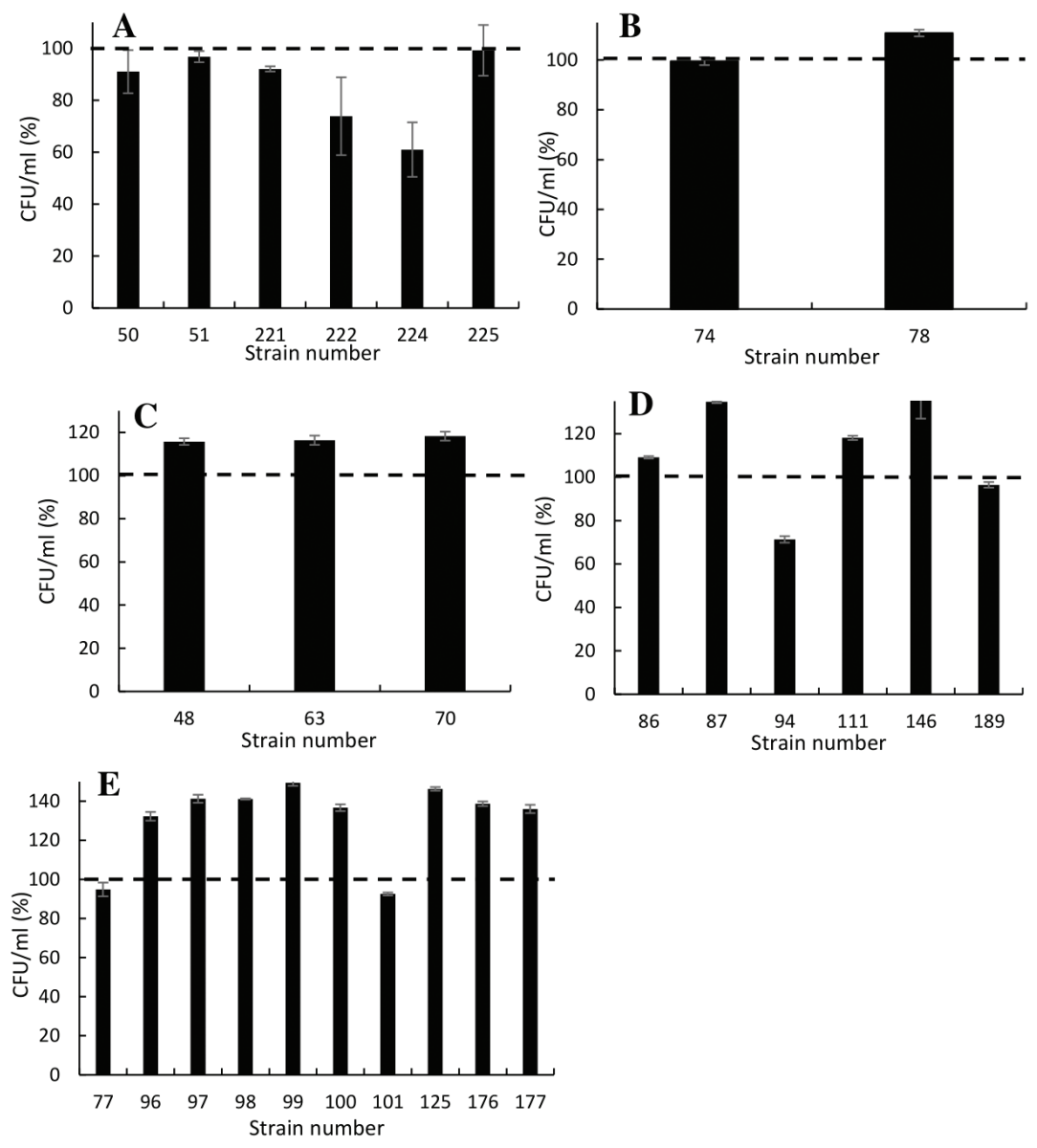

Fig. 3. Survival of the isolated 25 unique strains inoculated in liquid MRS supplemented with $0.4 \%$ phenol. Bars show the percentage of CFU/mL after $24 \mathrm{~h}$ of incubation; dash line indicates the starting CFU (normalized to $100 \%$ ). Graph A, B. coagulans; graph B, E. hirae; graph C, E. faecium; graph D, E. mundtii; and graph E, P. pentosaceus. 
(Fig. 3D). In this experiment, strains 222 and 224 showed a decrease of $30-40 \%$ in CFU $/ \mathrm{mL}$. Among other isolates, E. mundtii strains 87, 111, and 146 showed an average increase of $29 \%$ in CFU/mL (Fig. 3D). Strains belonging to P. pentosaceus (Fig. 3E) exhibited a high tolerance with a consistent level of CFU/mL before and after incubation for strains 77 and 101, while strains $96-100,125,176$, and 177 showed an average increase of $39 \%$ in CFU $/ \mathrm{mL}$.

\section{Bile tolerance}

B. coagulans (Fig. 4A) and E. hirae (Fig. 4B) strains showed a lowered viability after $24 \mathrm{~h}$ of incubation, followed by a larger drop in viability at $48 \mathrm{~h}$ of incubation with half of the strains completely depleted. Strains belonging to E. faecium showed a consistently high tolerance to $0.3 \%$ bile acid after $48 \mathrm{~h}$ of incubation, with an average decrease of $11 \%$ in $\log$ $\mathrm{CFU} / \mathrm{mL}$ (Fig. 4C). In the experiment, E. mundtii strains had inter-specific differences in response to bile acid. Strains 87, 94, and 146 (Fig. 4D) showed a high level of tolerance with a viable cell count close to the initial concentration; on the contrary, E. mundtii strains 86, 111, and 189 showed a low level of tolerance, where cells belonging to strain 189 was completely depleted after $48 \mathrm{~h}$ of incubation. Similar to the E. mundtii strains, P. pentosaceus (Fig. 4E) strains 77, $96,125,177$, and 176 had a small average increase, while strains 97, 99, 100, and 101 were completely depleted after incubation.

\section{Qualitative determination of BSH activity}

None of the strains belonging to $B$. coagulans and $P$. pentosaceus showed $\mathrm{BSH}$ activity, while all strains from E. faecium and E. mundtii showed BSH activity. Among E. hirae, we found both BSH-negative and BSH-positive strains (Table 2).

\section{Antibiotic resistance}

From the 11 screened Enterococcus strains, four strains (strains 63, 94, 146, and 189) showed susceptibility to all tested antibiotics. Of the remaining strains, three showed resistance toward one or more of the tested antibiotics and four showed intermediate resistance to at least one of the tested antibiotics (Table 3 ).

\section{A}
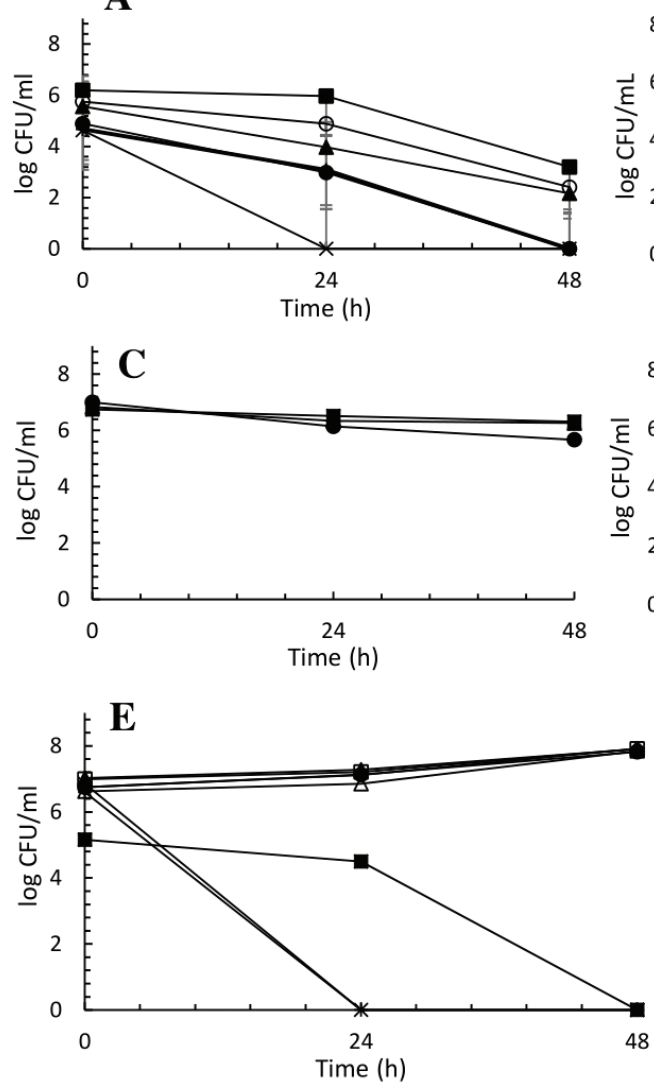

B
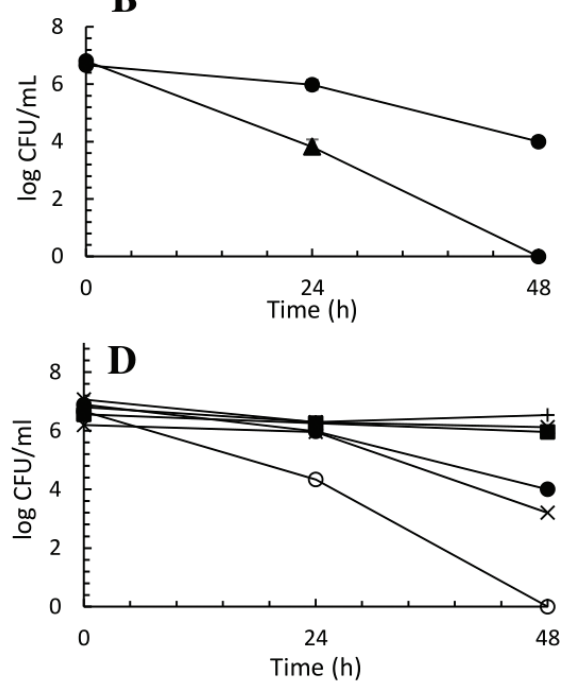

Fig. 4. Survival curves of 25 unique strains identified by MALDI-TOF, inoculated in liquid MRS supplemented with $0.3 \%$ bile. Graph A: B. coagulans; graph B: E. hirae; graph C: E. faecium; graph D: E. mundtii; and graph E: P. pentosaceus. Symbols correspond to different strain numbers. Graph A, B. coagulans: $50(\bullet), 51(\mathbf{\Delta}), 221(\mathbf{\bullet}), 222(\times), 224(+), 225(0)$; graph B, E. hirae:

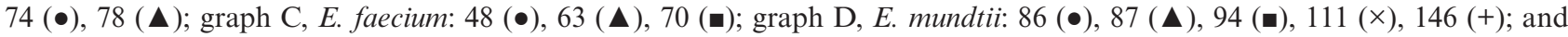
graph E, P. pentosaceus: $77(\bullet), 96(\mathbf{\Delta}), 97(\mathbf{\bullet}), 100(\times), 101(+), 125(\circ), 176(\Delta), 177(\square)$. 
Fermentation of fava bean flour medium by $P$. pentosaceus Each of the isolated and unique $P$. pentosaceus strains was able to grow in $6 \%$ fava bean medium without addition of carbon or any other nutrients. The strains showed a

Table 2. Bile salt hydrolase (BSH) activity assessed in all selected species

\begin{tabular}{lc}
\hline Species & BSH activity \\
\hline Bacillus coagulans & - \\
Enterococcus hirae 74 & - \\
Enterococcus hirae 78 & + \\
Enterococcus faecium & + \\
Enterococcus mundtii & + \\
Pediococcus pentosaceus & -
\end{tabular}

Differences within a species were only found in E. hirae.

Table 3. Resistance toward tested antibiotics measured as antibiotic disk diffusion zones (in millimeters)

\begin{tabular}{|c|c|c|c|c|}
\hline Isolate & Ampicillin & Trimethoprim & Vancomycin & Nitrofurantoin \\
\hline E. hirae 74 & $4(\mathrm{R})$ & $6(\mathrm{R})$ & $29(S)$ & $42(S)$ \\
\hline E. hirae 78 & $19(S)$ & $31(S)$ & $16(I)$ & $16(\mathrm{I})$ \\
\hline E. faecium 48 & $19(S)$ & $3(\mathrm{R})$ & $16(1)$ & $13(R)$ \\
\hline E. faecium 63 & $19(S)$ & $27(S)$ & $17(S)$ & II (S) \\
\hline E. faecium 70 & $19(S)$ & $27(S)$ & $17(S)$ & $16(\mathrm{I})$ \\
\hline E. mundtii 86 & $18(S)$ & $3(\mathrm{R})$ & $16(S)$ & I5 (I) \\
\hline E. mundtii 87 & $19(S)$ & $31(S)$ & $16(\mathrm{I})$ & $16(1)$ \\
\hline E. mundtii 94 & $21(S)$ & $4 \mathrm{I}(\mathrm{S})$ & $19(S)$ & $21(S)$ \\
\hline E. mundtii III & $19(S)$ & $34(S)$ & $16(\mathrm{I})$ & $17(S)$ \\
\hline E. mundtii I 46 & $23(S)$ & $32(S)$ & $17(S)$ & $19(S)$ \\
\hline E. mundtii 189 & $22(S)$ & $44(S)$ & $19(S)$ & $25(S)$ \\
\hline
\end{tabular}

Clinical and Laboratory Standards Institute (CLSI) interpretive categories are given in parentheses: S, susceptible; I, intermediate; and $\mathrm{R}$, resistant. growth pattern with an initial lag phase followed by exponential growth. During the exponential phase, the strains differed in generation time. Strains 97 and 100 had a generation time of approximately $1 \mathrm{~h}$, calculated by regression of data points between 2 and 8 h. Strains 77 and 96 had a generation time of approximately $2 \mathrm{~h}$ during the same time frame. Strain 176 had an intermediate generation time of $1.4 \mathrm{~h}$ under the given conditions. All strains reached a stationary phase after $8 \mathrm{~h}$ of incubation (Fig. 5).

\section{Co-fermentation of fava bean flour medium by P. pentosaceus 77 and P. kudriavzevii TYI322}

Yeast strain P. kudriavzeviiI TY1322 showed improved growth during co-fermentation with $P$. pentosaceus strain 77 compared to fermentation in monoculture. In both monoculture and co-fermentation, the yeast strain reached a stationary phase after $36 \mathrm{~h}$, but in co-fermentation, the cell count of $P$. kudriavzeviiI TY1322 was $124 \%$ higher compared to fermentation in monoculture (Fig. 6). The reduction of phytate in the fava bean medium by P. kudriavzevii TY1322 was significantly improved using a co-starter culture compared to fermentation monoculture (Fig. 7). After $48 \mathrm{~h}$ of fermentation, the phytate content in the medium fermented by $P$. kudriavzevii TY1322 in monoculture had decreased by $25 \%$. The phytate content in the medium fermented by a mixture of $P$. kudriavzevii TY1322 and $P$. pentosaceus 77 was reduced by $77 \%$. $P$. pentosaceus strain 77 was not able to reduce the phytate content by monoculture.

\section{Discussion}

There are strong arguments to change the food patterns in industrialized countries into more healthy and sustainable ones. Pulses, such as beans and lentils, are often described as a nutritious and sustainable protein source that could be used as alternatives to meat and dairy products.

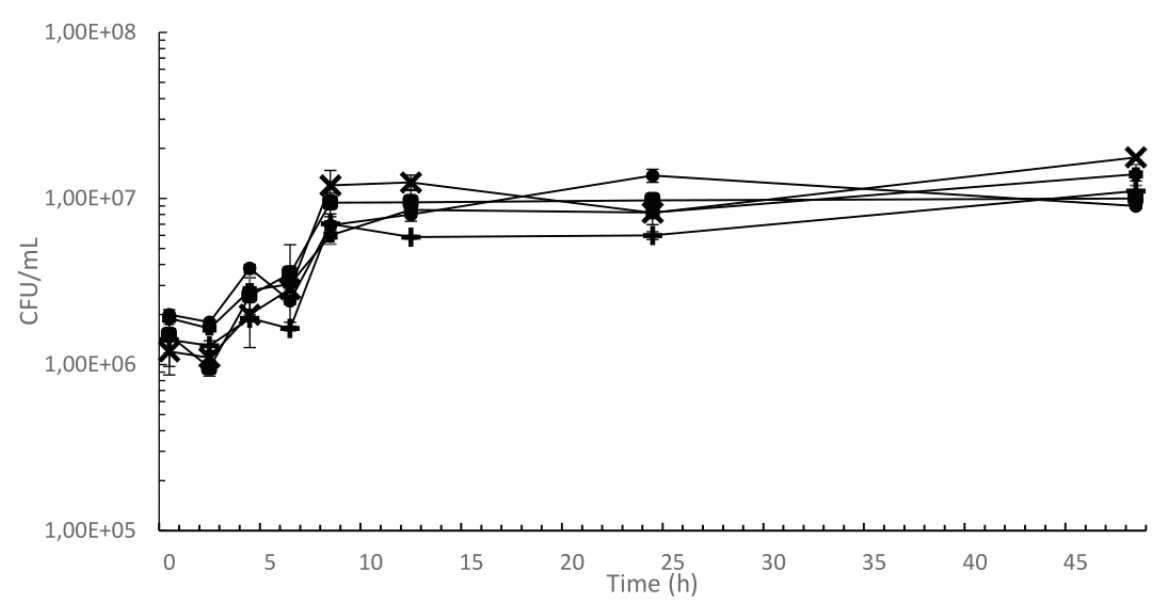

Fig. 5. Growth curve of selected $P$. pentosaceus strains in fava bean beverage without additional carbon sources. Strain numbers and their symbols are: $77(\bullet), 96(\boldsymbol{\Delta}), 97(\bullet), 100(\times), 176(+)$. Error bars represent standard errors. 


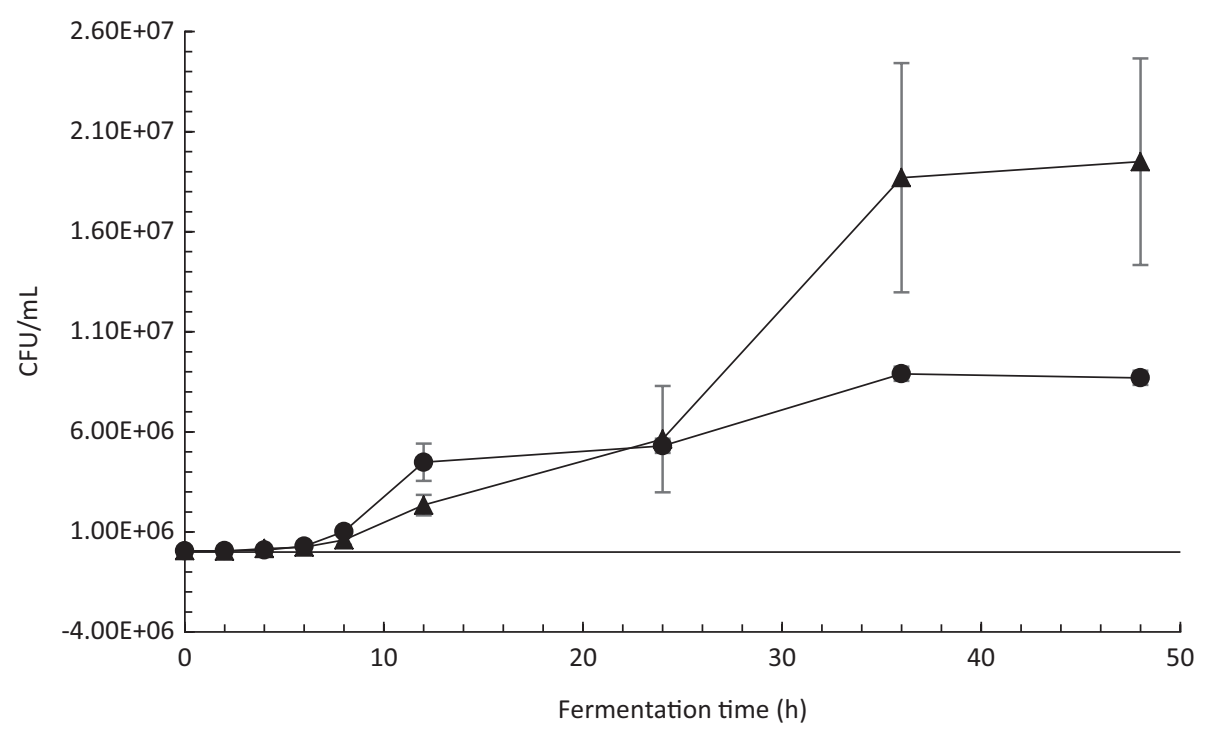

Fig. 6. Growth curve of P. kudriavzevii TY1322 in monoculture and in co-fermentation with P. pentosaceus strain 77 in fava bean medium. Symbols correspond to: . kudriavzevii TY1322 in monoculture $(\bullet)$ and in co-fermentation $(\boldsymbol{\Delta})$. Error bars represent standard errors.

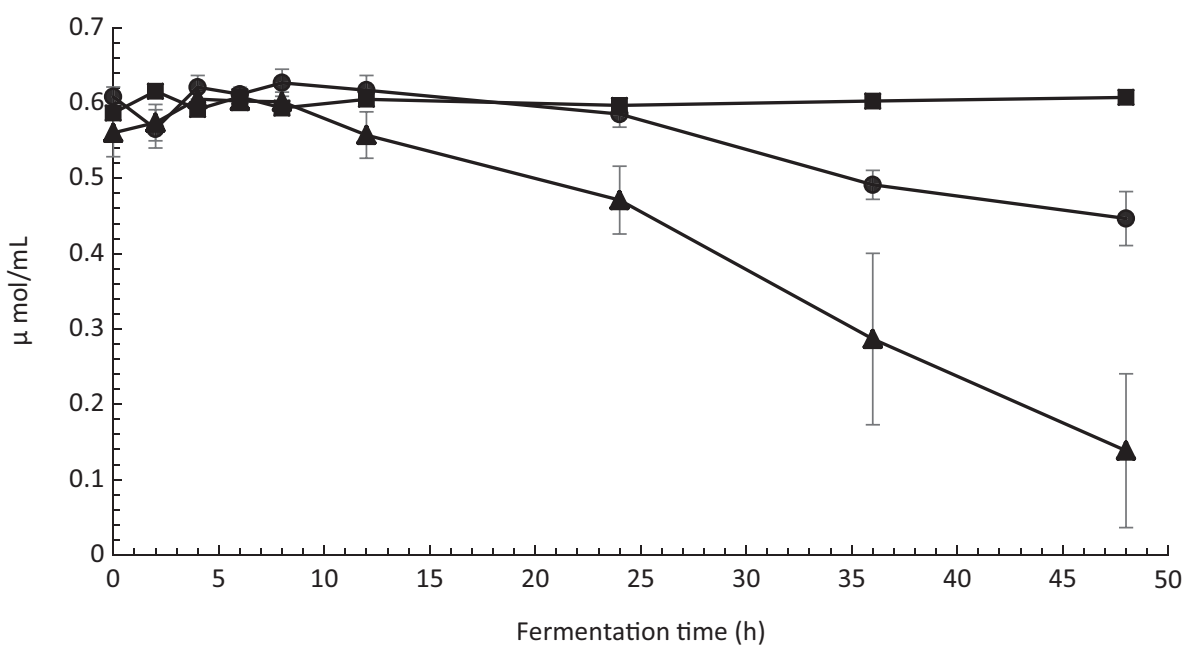

Fig. 7. Phytate content in fava bean medium fermented by P. kudriavzevii TY1322(•), P. pentosaceus strain 77 (घ), and by co-fermentation of both P. kudriavzevii and P. pentosaceus $(\mathbf{\Delta})$. Error bars represent standard errors.

Fermentation of legumes improve digestibility, natural preservation, and raise their nutritional value. Certain microbial strains may also be probiotic.

Today, despite the growing demand for dairy-free products, only very few fermented non-dairy products exist in the market worldwide, and few strains available commercially are aimed for the fermentation of plantbased foods (36).

In the present study, 116 isolates were obtained. Subsequently, they were sorted into 25 unique strains from three different genera as confirmed by rep-PCR and MALDI-TOF MS. The strains were identified as LAB, and closely related $B$. coagulans. Since $B$. coagulans is a spore forming bacteria, it can survive extreme conditions and has previously been suggested as an health-promoting ingredient (37). Owing to the spore forming ability, $B$. coagulans can survive human ingestion and reach the intestine where it germinates. In the intestine, it can contribute to positive effects such as production of short-chain fatty acids, promoting populations of beneficial bacteria (38), and aid in the digestion of protein and carbohydrates (39). The ability to increase protein bioavailability is of relevance in the ongoing protein shift toward more plant-based protein. Probiotic food products containing $B$. coagulans are currently sold to consumers commercially (37), and B. coagulans has received the Generally 
Recognized as Safe (GRAS) status and is reported as safe by the US Food and Drug Administration (FDA) (40).

The 25 unique strains were characterized in vitro in order to select candidate strains suitable for further investigation related to probiotic properties. Strains belonging to E. faecium, E. mundtii, and P. pentosaceus showed a high level of tolerance to $0.3 \%$ bile. Tolerance to bile and low $\mathrm{pH}$ is important to evaluate, since it indicates survival during the passage through the upper GIT, a necessary step for probiotic organisms delivered via food (41). Among the strains isolated in this study, strains 77, 100, 101, and 176 belonging to B. coagulans and P. pentosaceus have shown tolerance to $\mathrm{pH} 2.5$.

Tolerance to phenol was evaluated, since phenols may be formed in the gut by bacterial deamination of aromatic amino acids derived from dietary or endogenously produced proteins. Phenols and other products of protein degradation in the colon, such as ammonia, indoles, and amines, exert toxic effects in animal models and in vitro. These compounds are present in fecal samples and can exert bacteriostatic effects against some LAB (42).

We examined antibiotic resistance for Enterococcus strains because of the high occurrence of resistance within the Enterococcus genus, and because of the risk of pathogenicity. In the future studies, the presence of virulence genes should be addressed. Four of the Enterococcus strains (E. faecium 63 and E. mundtii 94, 146, and 189) were susceptible to all four tested antibiotics. Owing to their tolerance to salts and acids, Enterococcus strains are highly adapted to surviving in the GIT. Some Enterococcus strains have interesting properties relevant from food technology and medical perspective, such as the ability to produce multiple bacteriocins (43). Even though a few Enterococcus strains are currently commercialized as probiotics and used for treatment and prevention of certain diseases in humans and animals, these strains might originate from bovine feces used as manure in the fields, and may not be suitable to metabolize legumes $(43,44)$.

The ability to produce BSH enzyme, and thus detoxify bile salts, has often been included as a criterion for selection of probiotic strain (45). BSH catalyzes the deconjugation of bile salts to liberate free bile acids (46). Strains neither belonging to $P$. pentosaceus nor to $B$. coagulans were positive for BSH activity. On the contrary, strains belonging to E. mundtii and E. faecium were able to hydrolyze the bile salt TDCA along with E. hirae strain 78. The BSH activity indicates that the isolates not only survive toxicity of these salts but also de-conjugate these salts and may help in the intestinal colonization of cells and in reducing blood cholesterol level in the host (47).

We tested five selected strains belonging to $P$. pentosaceus for their ability to grow in a fava bean medium with no additional carbon, thus indicating their potential to ferment a bean beverage. Since strains belonging to $B$. coagulans are mainly of value as spores and not as fermenting organisms, and because of the uncertainties regarding Enterococcus during fermentation, these strains were excluded from fermentation. The five $P$. pentosaceus strains that were used for fermentation in this study had a similar growth pattern: an initial lag phase followed by exponential growth until $8 \mathrm{~h}$ of incubation, when the cells reached a stationary phase.

Finally, we showed the ability of one of the isolated $P$. pentosaceus strains to improve the reduction of phytate by the yeast strain $P$. kudriavzevii TY1322 in a fava bean medium. The origin of the parent strain TY13 and its properties regarding phytase production are described in the previous work by Hellström et al $(48,49,50)$. The symbiotic effect of $P$. pentosaceus strain 77 resulted in a $69 \%$ larger reduction compared to the monoculture and a $124 \%$ higher cell count of $P$. kudriavzevii TY1322 after $48 \mathrm{~h}$ of fermentation. This significantly improves the bioavailability of minerals such as iron and zinc, since phytate is a major inhibitor of iron and zinc absorption. This is especially important for individuals following a plantbased diet that does not contain dietary sources of heme iron.

$P$. pentosaceus strains have been previously isolated from legumes such as fava beans (51) and other unspecified beans (52). These findings are in line with our results. Strains of Pediococcus are currently used in the food industry as starter cultures for sausages, sauerkraut, cucumber, green bean, and soy sauce fermentation (53). Similarly, specific strains of Pediococcus are also used as probiotics for monogastric animals (54). The Pediococcus strains isolated in this study were able to use nutrients from fava beans, supporting their potential role as starters for further investigation and their possible use in the nondairy sector of food industry.

\section{Conclusion}

Some of the strains isolated can be of interest from the perspective of food technology as potential starters for fermentation of non-dairy legume-based products, and others may have potential to be probiotic candidate strains. Further investigation on probiotic properties are needed, such as adherence to gastric mucosa. Five strains showed tolerance to all three conditions tested (survival through GIT, tolerance to bile and phenol, and low $\mathrm{pH}$ ). Three of these strains belong to B. coagulans (strains 51, 221 , and 225), and the other two belong to $P$. pentosaceus (strains 77 and 176), which are of interest regarding fermentation, presenting an interesting possibility to produce a combined fermented and probiotic dairy-free food product. The $P$. pentosaceus strains that were isolated in the present study were suitable for the fermentation of a 
legume beverage. One of the $P$. pentosaceus strains was also able to improve the reduction of phytate by the phytase producing yeast strain $P$. kudriavzevii TY1322 during co-fermentation as well as increase the final cell count of yeast strain. This is a positive indication that new and improved fermented non-dairy products could be produced for commercial use and that strains with probiotic potential can be selected.

\section{Acknowledgements}

Financial support from Ekhagastiftelsen and C. F. Lundströms stiftelse are greatly acknowledged. The authors thank Gotland Ölands Trädgårdsprodukter for providing samples and access to fields.

The authors also thank Viviana Moroso (MSc, PhD) of MV Medical Writing (Luleå, Sweden) for providing medical writing support for this manuscript in accordance with the Good Publication Practice (GPP3) guidelines.

\section{Conflict of interest and funding}

The authors declare no conflicts of interest.

\section{Authors' contributions}

Conceptualization, Cecilia Mayer Labba; Methodology, Fei Sjöberg, Thomas Andlid, and Åsa Lindgren; Laboratory work and data analysis, Cecilia Mayer Labba and Åsa Lindgren; Laboratory supervision, Fei Sjöberg and Thomas Andlid; Supervision, AnnSofie Sandberg; writing of original draft, Cecilia Mayer Labba; and writing, reviewing, and editing, Thomas Andlid, Åsa Lindgren, Ann-Sofie Sandberg, and Fei Sjöberg.

\section{References}

1. Rebello CJ, Greenway FL, Finley JW. Whole grains and pulses: a comparison of the nutritional and health benefits. J Agric Food Chem 2014; 62: 7029-49. doi: 10.1021/jf500932z

2. Stagnari F, Maggio A, Galieni A, Pisante M. Multiple benefits of legumes for agriculture sustainability: an overview. Chem Biol Technol Agric 2017; 4: 2. doi: 10.1186/s40538-0160085-1

3. Gan R-Y, Li HB, Gunaratne A, Sui ZQ, Corke H. Effects of fermented edible seeds and their products on human health: bioactive components and bioactivities. Compr Rev Food Sci. Food Safety (CRFSFS) 2017; 16(3): 489-531. doi: 10.1111/1541-4337.12257

4. Tiwari BK, Gowen A, Mckenna B. Pulse foods. Processing, quality and nutraceutical applications. Cambridge, MA: Elsevier; 2011.

5. Hou J-W, Yu R-C, Chou C-C. Changes in some components of soymilk during fermentation with bifidobacteria. Food Res Int 2000; 33: 393-7. doi: 10.1016/s0963-9969(00)00061-2

6. Yang H, Zhang L, Ziao G, Feng J, Zhou H, Huang F. Changes in some nutritional components of soymilk during fermentation by the cukinary and medicinal mushroom Grifola frondosa. Food Sci Tech (LWT) 2015; 62: 468-73. doi: 10.1016/j. lwt.2014.05.027
7. Gu Q, Zhang C, Song D, Li P, Zhu X. Enhancing vitamin B12 content in soy-yogurt by Lactobacillus reuteri. Int J Food Sci Tech (IJFST) 2015; 206: 56-9. doi: 10.1016/j. ijfoodmicro.2015.04.033

8. Desai A, Small D, Mcgill AEJ, Shah NP. Metabolism of raffinose and stachyose in reconstituted skil milk and of n-hexanal and pentanal in soymilk by bifidobacteria. Biosci Microfl 2002; 21: 245-50. doi: 10.12938/bifidus1996.21.245

9. Sandberg A-S. Developing functional ingredient. A case study of pea protein. Abington: Woodhead; 2011.

10. Wang Y-C, Yu R-C, Yang H-Y, Chou C-C. Sugar and acid contents in soymilk fermented with lactic acid bacteria alone or simultaneously with bifidobacteria. Food Microbiol 2002; 20: 333-8. doi: 10.1016/s0740-0020(02)00125-9

11. Fredrikson M, Andlid T, Haikara A, Sandberg A-S. Phytate degradation by micro-organisms in synthetic media and pea flour. J Appl Microbiol 2002; 93: 197-204. doi: 10.1046/j.1365-2672.2002.01676.x

12. Sharma A, Kumari S, Wongputtisin P, Nout MJR, Sarkar PK. Optimization of soybean processing into kinema, a Bacillusfermented alkaline food with respect to a minimum level of antinutrients. J Appl Microbiol 2015; 119: 162-76. doi: 10.1111/ jam. 12826

13. Hickisch A, Beer R, Vogel RF, Toelstede S. Influence of lupin-based milk alternative heat treatment and exopolysaccharide-producing lactic acid bacteria on the physical characteristics of lupin-based yogurt alternatives. Food Res Int 2016; 84: 180-8. doi: 10.1016/j.foodres.2016.03.037

14. Reale A, Konietzny U, Coppola R, Sorrentino E, Greiner R. The importance of lactic acid bacteria for phytate degradation during cereal dough fermentation. J Agric Food Chem 2007; 55: 2993-7. doi: 10.1021/jf063507n

15. Narvhus J, Gadaga $H$. The role of interaction between yeasts and lactic acid bacteria in African fermented milks: a review. Int J Food Microbiol 2003; 86: 51-60. doi: 10.1016/ s0168-1605(03)00247-2

16. Nyambane B, Thari WM, Wangoh J, Njage PM. Lactic acid bacteria and yeasts involved in the fermentation of amabere amaruranu, a Kenyan fermented milk. Food Sci Nutr 2014; 2: 692-9. doi: 10.1002/fsn3.162

17. Plessas S, Fisher A, Koureta K, Psarianos C, Nigam P, Koutinas AA. Application of Kluyveromyces marxianus, Lactobacillus delbrueckii ssp. bulgaricus and L. helveticus for sour dough bread making. Food Chem 2008; 106: 985-90. doi: 10.1016/j. foodchem.2007.07.012

18. FAO/WHO. Report of a Joint FAO/WHO expert consultation on evaluation of health nutritional properties of probiotics in food including powder mild and live lactic acid bacteria. London, ON: World Health Organization and Food and Agriculture Organization of the United Nations; 2001.

19. Bukowska H, Pieczul-Mroz J, Jastrzebska M, Chelstowski K, Naruszewicz M. Decrease in fibrinogen and LDL-cholesterol levels upon supplementation of diet with Lactobacillus plantarum in subjects with moderately elevated cholesterol. Atherosclerosis 1998; 137: 437-8. doi: 10.1016/s0021-9150(97)89666-9

20. Niedzielin K, Kordecki H, Birkenfeld B. A controlled, double-blind, randomized study on the efficacy of Lactobacillus plantarum 299V in patients with irritable bowel syndrome. Eur J Gastroenterol Hepatol 2001; 13: 1143-7. doi: 10.1097/00042737-200110000-00004

21. Nobaek S, Johansson ML, Molin G, Ahrne A, Jeppsson B. Alteration of intestinal microflora is associated with reduction in abdominal bloating and pain in patients with 
irritable bowel syndrome. Am J Gastroenterol 2000; 95: 1231-8. doi: 10.1111/j.1572-0241.2000.02015.x

22. Hill C, Guarner F, Reid G, Gibson GR, Merenstein DJ, Pot B, et al. The International Scientific Association for Probiotics and Prebiotics consensus statement on the scope and appropriate use of the term probiotic. Nat Rev Gastroenterol Hepatol 2014; 11: 505-14. doi: 10.1038/nrgastro.2014.66

23. Guarner G, Khan A, Garisch J, Eliakim R, Gangl A, Thomson A, et al. World gastroenterology organisation global guidelines: probiotics and prebiotics October 2011. J Clin Gastroenterol 2012; 46: 468-81. doi: 10.1097/mcg.0b013e3182549092

24. Valdes AM, Walter J, Segal E, Spector TD. Role of the gut microbiota in nutrition and health. BMJ 2018; 361: k2179. doi: 10.1136/bmj.k2179

25. Guarner F, Schaafsma GJ. Probiotics. Int J Food Microbiol 2008; 39: 237-8. doi: 10.1016/S0168-1605(97)00136-0

26. Sjöberg F, Nowrouzian F, Rangel İ, Hannoun C, Moore E, Adlerberth I, et al. Comparison between terminal-restriction fragment length polymorphism (T-RFLP) and quantitative culture for analysis of infants' gut microbiota. J Microbiol Methods 2013; 94: 37-46. doi: 10.1016/j.mimet.2013.04.002

27. Versalovic J, Schneider M, Bruijn FJD, Lupski JR. Genomic fingerprinting of bacteria using repetitive sequence-based polymerase chain reaction. Methods Mol Cell Biol 1994; 5: 25-40.

28. Verdenelli MC, Ghelfi F, Silvi S, Orpianesi C, Cecchini C, Cresci A. Probiotic properties of Lactobacillus rhamnosus and Lactobacillus paracasei isolated from human faeces. Eur J Nutr 2009; 48: 355-63. doi: 10.1007/s00394-009-0021-2

29. Xanthopoulos V, Litopoulou-Tzanetaki E, Tzanetakis N. Characterization of Lactobacillus isolates from infant faeces as dietary adjuncts. Food Microbiol 2000; 17: 205-15. doi: 10.1006/ fmic. 1999.0300

30. Gilliland SE, Walker DK. Factors to consider when selecting a culture of Lactobacillus acidophilus as a dietary adjunct to produce a hypocholesterolemic effect in humans. J Dariy Sci 1990; 73: 905-11. doi: 10.3168/jds.s0022-0302(90)78747-4

31. Dashkevicz MP, Feighner SD. Development of a differential medium for bile salt hydrolase-active Lactobacillus spp. Appl Environ Microbiol 1989; 55: 11-16. doi: 10.1128/ aem.55.1.11-16.1989

32. Clinical and Laboratory Standards Institute (CLSI) Performance standards for antimicrobial disk susceptibility tests; approved standard. Wayne, PA: CLSI; 2015.

33. Qvirist L, Vorontsov E, VeideVilg J, Andlid T. Strain improvement of Pichia kudriavzevii TY13 for raised phytase production and reduced phosphate repression. Microb Biotechnol 2017; 10: 341-53. doi: 10.1111/1751-7915.12427

34. Carlsson NG, Bergman EL, Skoglund E, Hasselblad K, Sandberg AS. Rapid analysis of inositol phosphates. J Agric Food Chem 2001; 49: 1695-701. doi: 10.1021/jf000861r

35. Maluping RP, Ravelo C, Lavilla-Pitogo CR, Krovacek K, Romalde JL. Molecular typing of Vibrio parahaemolyticus strains isolated from the Philippines by PCR-based methods. J Appl Microbiol 2005; 99: 383-91. doi: 10.1111/j.13652672.2005.02571.x

36. Granato D, Branco GF, Nazzaro F, Cruz AG, Faria JAF. Functional foods and non-dairy probiotic food development: trends, concepts, and products. Compr Rev Food Sci Food Saf 2010; 9(3): 292-302. doi: 10.1111/j.1541-4337.2010.00110.x

37. Elshaghabee FMF, Rokana N, Gulhane RD, Sharma C, Panwar H. Bacillus as potential probiotics: status, concerns, and future perspectives. Front Microbiol 2017; 8: 1490. doi: 10.3389/ fmicb. 2017.01490

38. Nyangale EP, Farmer S, Keller D, Chernoff D, Gibson GR. Effect of prebiotics on the fecal microbiota of elderly volunteers after dietary supplementation of Bacillus coagulans GBI-30, 6086. Anaerobe 2014; 30: 75-81. doi: 10.1016/j.anaerobe.2014.09.002

39. Maathuis A, Keller D, Farmer S. Survival and metabolic activity of the Ganeden BC30 strain of Bacillus coagulans in a dynamic in vitro model of the stomach and small intestine. Benef Microb 2010; 1: 31-6. doi: 10.3920/bm2009.0009

40. Hazards EPOB. Scientific opinion on the maintenance of the list of QPS biological agents intentionally added to food and feed (2013 update). EFSA J 2013; 11: 3449. doi: 10.2903/j. efsa.2013.3449

41. Gueimonde M, Salminen S. New methods for selecting and evaluating probiotics. Digest Liver Dis 2006; 38: S242-7. doi: 10.1016/s1590-8658(07)60003-6

42. Hughes R, Magee EAM, Bingham S. Protein degradation in the large intestine: relevance to colorectal cancer. Curr Issues Interest Microbiol 2000; 1: 51-8.

43. Hanchi H, Mottawea W, Sebei K, Hammami R. The genus Enterococcus: between probiotic potential and safety concerns - an update. Front Microbiol 2018; 9: 1791. doi: 10.3389/ fmicb.2018.01791

44. Franz CMAP, Holzapfel WH, Stiles ME. Enterococci at the crossroads of food safety? Int J Food Microbiol 1999; 47: 1-24. doi: 10.1016/s0168-1605(99)00007-0

45. Noriega L, Cuevas I, Margolles A, Reyes-Gavilan CGDL. Deconjugation and bile salts hydrolase activity by Bifidobacterium strains with acquired resistance to bile. Int Dairy J 2006; 16: 850-5. doi: 10.1016/j.idairyj.2005.09.008

46. Gilliland SE, Speck ML. Deconjugation of bile acids by intestinal lactobacilli. Appl Environ Microbiol 1977; 33: 15-18. doi: 10.1128/aem.33.1.15-18.1977

47. Toit MD, Franz CM, Dicks LM, Schillinger U, Harberer P, Warlies B, et al. Characterization and selection of probiotic lactobacilli for a preliminary mini-pig feeding trial and their effect on serum cholesterol levels, feces $\mathrm{pH}$, and feces moisture content. IJFST 1998; 40: 93-104. doi: 10.1016/ s0168-1605(98)00024-5

48. Hellström A, Qvirist L, Svanberg U, Veide Vilg J \& Andlid T. Secretion of non-cell-bound phytase by the yeast Pichia kudriavzevii TY13. Journal of Applied Microbiology, 2015; 118, 1126-1136. doi: 10.1111/jam.12767

49. Hellström AM, Almgren A, Carlsson N-G, Svanberg U \& Andlid TA. Degradation of phytate by Pichia kudriavzevii TY13 and Hanseniaspora guilliermondii TY14 in Tanzanian togwa. International Journal of Food Microbiology, 2012; 153, 73-77. doi: 10.1016/j.ijfoodmicro.2011.10.018

50. Hellström AM, Vázques-Juárez R, Svanberg U \& Andlid TA. Biodiversity and phytase capacity of yeasts isolated from Tanzanian togwa. International Journal of Food Microbiology, 2010; 136, 352-358. doi: 10.1016/j.ijfoodmicro.2009.10.011

51. Rizzello CG, Coda R, Wang Y, Verni M, Kajala I, Katina K, et al. Characterization of indigenous Pediococcus pentosaceus, Leuconostoc kimchii, Weissella cibaria and Weissella confusa for Faba bean bioprocessing. Int J Food Microbiol 2019; 302: 24-34. doi: 10.1016/j.ijfoodmicro.2018.08.014

52. Venkateshwari S, Halami PM, Vijayendra SVN. Characterisation of the heat-stable bacteriocin-producing and vancomycin-sensitive Pediococcus pentosaceus CFR B19 isolated from beans. Benef Microb 2009; 1: 159-64. 
53. Simpson WJ, Taguchi H. The genus Pediococcus, with notes on the genera Tetratogenococcus and Aerococcus. In: Wood BJB, Holzapfel WH, eds. The genera of lactic acid bacteria. Boston, MA: Springer; 1995, pp. 125-172.

54. Chaucheyras-Durand F, Durand H. Probiotics in animal nutirtion and health. Benef Microb 2009; 1: 3-9. doi: 10.3920/ bm2008.1002

\section{*Inger-Cecilia Mayer Labba}

Division of Food and Nutrition Science,

Department of Biology and Biological Engineering

Chalmers University of Technology

Kemivägen 10, SE-412 96Gothenburg, Sweden

Email: cecilia.mayer.labba@chalmers.se 